TRANSACTIONS OF THE

AMERICAN MATHEMATICAL SOCIETY

Volume 353, Number 8, Pages 3097-3114

S 0002-9947(01)02686-1

Article electronically published on March 12, 2001

\title{
COHOMOLOGY OF PROJECTIVE SPACE SEEN BY RESIDUAL COMPLEX
}

\author{
I-CHIAU HUANG
}

\begin{abstract}
A subcomplex of a residual complex on projective space is constructed for computing the cohomology modules of locally free sheaves. A constructive new proof of the Bott formula is given by explicitly exhibiting bases for the cohomology modules.
\end{abstract}

\section{INTRODUCTION}

This paper is about the cohomology structure of locally free sheaves on a projective space. It is a part of the program for rendering concrete realizations of Grothendieck duality and finding applications of such realizations. In the paper, we propose a new method to organize cohomology data using residual complexes.

Let $\kappa$ be a field. The cohomology modules of quasi-coherent sheaves on the $n$-dimensional projective space $\mathbb{P}^{n}$ over $\kappa$ are usually calculated using Čech cohomology. The need for Čech cohomology is supported by the common belief that injective resolutions of a given sheaf of modules are inaccessible from the computational point of view. Our philosophy about this belief is that, for sheaves of modules related to duality theory, some injective complexes can be explicitly constructed and give rise directly to cohomology information. For instance, the Gorenstein property of the ring $\kappa\left[\left[X^{t_{1}}, \cdots, X^{t_{n}}\right]\right]$ is characterized by a value semigroup via a complex of injective modules

$$
\cdots \rightarrow 0 \rightarrow \kappa((X)) \rightarrow \operatorname{Hom}_{\kappa}^{c}\left(\kappa\left[\left[X^{t_{1}}, \cdots, X^{t_{n}}\right]\right], \kappa\right) \rightarrow 0 \rightarrow \cdots
$$

see [7]. In 9], some classical theorems on the projective plane, such as the Newton theorem and the Cayley-Bacharach theorem, are obtained from a complex of injective sheaves of modules. The complexes used in the above-cited works are called residual complexes, and they become subtle in higher dimensional projective spaces. In this paper, our philosophy on injective resolutions is emphasized by illustrating how the cohomology modules of locally free sheaves on $\mathbb{P}^{n}$ are determined by the structure of a residual complex on $\mathbb{P}^{n}$.

We recall the definition of residual complexes. Let $\mathcal{X}$ be a locally Noetherian scheme. A residual complex $\mathcal{M}^{\bullet}$ on $\mathcal{X}$ is a complex of quasi-coherent injective $\mathcal{O}_{\mathcal{X}}$ modules, bounded below, with coherent cohomology sheaves, and such that there

Received by the editors January 10, 2000.

2000 Mathematics Subject Classification. Primary 14F05, 14F10; Secondary 13 N05.

Key words and phrases. Bott formula, injective resolution, residual complex.

(C)2001 American Mathematical Society 
is an isomorphism

$$
\bigoplus_{i \in \mathbb{Z}} \mathcal{M}^{i} \simeq \bigoplus_{\mathfrak{p} \in \mathcal{X}} J(\mathfrak{p})
$$

where $J(\mathfrak{p})$ is the quasi-coherent $\mathcal{O}_{\mathcal{X}}$-module which is the constant sheaf $M(\mathfrak{p})$, a given injective hull of the residue field $\kappa(\mathfrak{p})$ over the local $\operatorname{ring} \mathcal{O}_{\mathcal{X}, \mathfrak{p}}$, on $\{\mathfrak{p}\}^{-}$, and 0 elsewhere [3, page 304]. The importance of residual complexes is shown in [3], which lays the foundation of Grothendieck duality using the language of derived categories and derived functors. However, the subtlety of the coboundary maps

$$
\mathcal{M}^{i} \rightarrow \mathcal{M}^{i+1} \quad(i \in \mathbb{Z})
$$

of residual complexes, already mentioned by Grothendieck [2, page 113], is not fully treated in [3]. Fortunately, residual complexes and their properties are now better understood in concrete terms, thanks to [5], 6], 8], 10], 11], 12, 13, 14, [15, [16 and others. With success in concrete realizations of Grothendieck duality, it is desirable to see what information on schemes one gets from these concrete aspects of duality.

This paper focuses on cohomology modules of locally free sheaves of modules on $\mathbb{P}^{n}$. The next section sketches a residual complex $J^{\bullet}$ on $\mathbb{P}^{n}$ which is a simplified version of the residual complex constructed in [5]. As $J^{\bullet}$ is an injective resolution of $\mathcal{O}_{\mathbb{P}^{n}}(-n-1)$, the complex $J^{\bullet} \otimes \mathcal{F}(n+1)$ provides an injective resolution for any locally free sheaf of modules $\mathcal{F}$ on $\mathbb{P}^{n}$. In section 3 , we investigate the structure of $J^{\bullet} \otimes \mathcal{F}(n+1)$. We construct a subcomplex $\mathcal{F}^{(\bullet)}$ of $J^{\bullet} \otimes \mathcal{F}(n+1)$ which gives rise to the cohomology modules of $\mathcal{F}$. In the last section, we explicitly give a basis for $\mathrm{H}^{q}\left(\mathbb{P}^{n}, \Omega_{\mathbb{P}^{n} / \kappa}^{p}(m)\right)$ by working on the complex $\Omega_{\mathbb{P}^{n} / \kappa}^{p}(m)^{(\bullet)}$ to illustrate that $\mathcal{F}^{(\bullet)}$ is indeed suitable for computing the cohomology modules of $\mathcal{F}$. More precisely, we give a basis $\left\{\omega_{t_{0} \cdots t_{n-q} \cdots t_{n}}^{j_{1} \cdots j_{p}}\right\}$ for $\Omega_{\mathbb{P}^{n} / \kappa}^{p}(m)^{(q)}$ and explicitly describe coboundary maps

$$
d^{(q)}: \Omega_{\mathbb{P}^{n} / \kappa}^{p}(m)^{(q)} \rightarrow \Omega_{\mathbb{P}^{n} / \kappa}^{p}(m)^{(q+1)}
$$

in terms of operators $\frac{X_{\ell}}{X_{n-q}}$ on $\omega_{t_{0} \cdots t_{n-q} \cdots t_{n}}^{j_{1} \cdots j_{p}}$. We will prove the following facts:

1. $\mathrm{H}^{0}\left(\mathbb{P}^{n}, \Omega_{\mathbb{P}^{n} / \kappa}^{p}(m)\right)$ has a basis consisting of

$$
\sum_{\ell=0}^{p}(-1)^{\ell+1} \frac{X_{j_{\ell}}}{X_{n}} \omega_{t_{0} \cdots t_{n-i} 0 \cdots \underline{0}}^{j_{j} \cdots \hat{j}_{\ell} \cdots j_{p}}
$$

satisfying

- $0 \leq i \leq n-p$,

- $0 \leq j_{0}<\cdots<j_{p}=n-i$,

- $t_{0}, \cdots, t_{n-i} \geq 0$

- $t_{0}+\cdots+t_{n-i}=m-p-1$.

(Note that, to satisfy these conditions, $p$ has to be less than $m$.)

2. For $0<q<n, \mathrm{H}^{q}\left(\mathbb{P}^{n}, \Omega_{\mathbb{P}^{n} / \kappa}^{p}(m)\right)$ has a basis consisting of

$$
\omega_{0 \cdots 0}^{(n-q+1)(n-q+2) \cdots n} \cdots(-1) \cdots
$$

(For this element to be well-defined, $p$ has to equal $q$ and $m$ has to vanish.)

3. $\mathrm{H}^{n}\left(\mathbb{P}^{n}, \Omega_{\mathbb{P}^{n} / \kappa}^{p}(m)\right)$ has a basis consisting of

$$
\omega_{(-1) \cdots(-1) t_{i} \cdots t_{n}}^{12 \cdots i j_{i+1} \cdots j_{p}}
$$




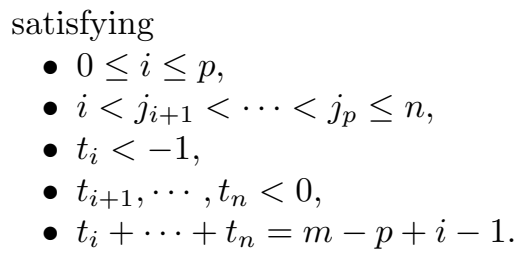

(Note that, to satisfy these conditions, $m$ has to be less than $p-n$.)

Counting the cardinality of these bases (see Corollary 4.4 and Corollary 4.9 ), the Bott formula is recovered.

\section{Residual Complex on Projective Space}

As we work on a projective space $\mathbb{P}^{n}=\operatorname{Proj}\left(\kappa\left[X_{0}, X_{1}, \cdots, X_{n}\right]\right)$ over $\kappa$, the approach in [5] to residual complexes can be simplified. In particular, a canonical injective hull of the residue field of a point on $\mathbb{P}^{n}$ can be constructed without the identifying process in [5, section 2].

For each point $\mathfrak{p} \in \mathbb{P}^{n}$, the $\mathcal{O}_{\mathbb{P}^{n}, \mathfrak{p}}$-module

$$
M_{\mathbb{P} n}(\mathfrak{p}):=\mathrm{H}_{\mathfrak{m}_{\mathfrak{p}}}^{d}\left(\Omega_{\mathcal{O}^{n}, \mathfrak{p} / \kappa}^{n}\right),
$$

where $d$ is the height of $\mathfrak{p}$ and $\mathfrak{m}_{\mathfrak{p}}$ is the maximal ideal of $\mathcal{O}_{\mathbb{P}^{n}, \mathfrak{p}}$, is an injective hull of the residue field of

$$
\kappa\left[\frac{X_{0}}{X_{i}}, \cdots, \frac{X_{i-1}}{X_{i}}, \frac{X_{i+1}}{X_{i}}, \cdots, \frac{X_{n}}{X_{i}}\right]_{\mathfrak{p}} .
$$

Let $J_{\mathbb{P}^{n}}(\mathfrak{p})$ be the constant sheaf which is $M_{\mathbb{P}^{n}}(\mathfrak{p})$ on $\{\mathfrak{p}\}^{-}$, and 0 elsewhere. We write $M_{\mathbb{P} n}(\mathfrak{p})$ and $J_{\mathbb{P}^{n}}(\mathfrak{p})$ simply as $M(\mathfrak{p})$ and $J(\mathfrak{p})$, if it is clear from the context that we are working on $\mathbb{P}^{n}$. Cover $\mathbb{P}^{n}$ by affine open subsets

$$
V_{i}=\operatorname{Spec} \kappa\left[\frac{X_{0}}{X_{i}}, \cdots, \frac{X_{i-1}}{X_{i}}, \frac{X_{i+1}}{X_{i}}, \cdots, \frac{X_{n}}{X_{i}}\right] .
$$

If $\mathfrak{p}$ is contained in some $V_{i}$, then there is a canonical isomorphism

$$
M(\mathfrak{p}) \simeq \mathrm{H}_{\mathfrak{m}_{\mathfrak{p}}}^{d}\left(\Omega_{\kappa\left[\frac{X_{0}}{X_{i}}, \cdots, \frac{X_{i-1}}{X_{i}}, \frac{X_{i+1}}{X_{i}}, \cdots, \frac{X_{n}}{X_{i}}\right]_{\mathfrak{p}} / \kappa}\right) .
$$

By identifying the above two modules, elements in $M(\mathfrak{p})$ can be described by generalized fractions

$$
\left[\begin{array}{c}
\omega \\
f_{1}^{t_{1}}, \cdots, f_{d}^{t_{d}}
\end{array}\right]
$$

where

$$
\omega \in \Omega_{\kappa\left[\frac{X_{0}}{X_{i}}, \cdots, \frac{X_{i-1}}{X_{i}}, \frac{X_{i+1}}{X_{i}}, \cdots, \frac{X_{n}}{X_{i}}\right]_{\mathfrak{p}} / \kappa}, \quad t_{1}, \cdots, t_{d} \geq 1,
$$

and $f_{1}, \cdots, f_{d}$ is a system of parameters of $\kappa\left[\frac{X_{0}}{X_{i}}, \cdots, \frac{X_{i-1}}{X_{i}}, \frac{X_{i+1}}{X_{i}}, \cdots, \frac{X_{n}}{X_{i}}\right]_{\mathfrak{p}}$. The reader is referred to [6. Chapter 2] for the definition and properties of generalized fractions. In this paper, we allow $t_{\ell}$ to be any integer: By convention,

$$
\left[\begin{array}{c}
\omega \\
f_{1}^{t_{1}}, \cdots, f_{d}^{t_{d}}
\end{array}\right]=0
$$

if some $t_{\ell} \leq 0$. 
Example 1. For each $1 \leq i \leq n$, let $\mathfrak{p}$ be the generic point of the closed subscheme $\mathbb{P}^{i}:=\operatorname{Proj}\left(\kappa\left[X_{0}, \cdots, X_{i}\right]\right)$ of $\mathbb{P}^{n}$. The elements of $M(\mathfrak{p})$ are sums of the elements of the form

$$
\left[\begin{array}{c}
\frac{h}{g} d \frac{X_{1}}{X_{0}} \wedge \cdots \wedge d \frac{X_{n}}{X_{0}} \\
\left(\frac{X_{i+1}}{X_{0}}\right)^{t_{i+1}}, \cdots,\left(\frac{X_{n}}{X_{0}}\right)^{t_{n}}
\end{array}\right]
$$

where $g, h \in \kappa\left[\frac{X_{1}}{X_{0}}, \cdots, \frac{X_{i}}{X_{0}}\right]$ and $t_{i+1}, \cdots, t_{n} \geq 1$.

Example 2. Let $\mathfrak{p}$ be a point in $\mathbb{P}^{i} \backslash \mathbb{P}^{i-1}$ for some $1 \leq i \leq n$. As a prime ideal of $\kappa\left[\frac{X_{0}}{X_{i}}, \cdots, \frac{X_{n}}{X_{i}}\right], \mathfrak{p}$ is generated by a prime ideal $\mathfrak{p}^{\prime}$ of $\kappa\left[\frac{X_{0}}{X_{i}}, \cdots, \frac{X_{i-1}}{X_{i}}\right]$ and $\frac{X_{i+1}}{X_{i}}, \cdots, \frac{X_{n}}{X_{i}}$. The elements of $M(\mathfrak{p})$ are sums of the elements of the form

$$
\left[\begin{array}{c}
\frac{h}{g} d \frac{X_{0}}{X_{i}} \wedge \cdots \wedge d \frac{X_{i-1}}{X_{i}} \wedge d \frac{X_{i+1}}{X_{i}} \wedge \cdots \wedge d \frac{X_{n}}{X_{i}} \\
f_{1}, \cdots, f_{\ell},\left(\frac{X_{i+1}}{X_{i}}\right)^{t_{i+1}}, \cdots,\left(\frac{X_{n}}{X_{i}}\right)^{t_{n}}
\end{array}\right]
$$

where

$h \in \kappa\left[\frac{X_{0}}{X_{i}}, \cdots, \frac{X_{i-1}}{X_{i}}\right], g \in \kappa\left[\frac{X_{0}}{X_{i}}, \cdots, \frac{X_{i-1}}{X_{i}}\right] \backslash \mathfrak{p}^{\prime}, f_{1}, \cdots, f_{\ell} \in \kappa\left[\frac{X_{0}}{X_{i}}, \cdots, \frac{X_{i-1}}{X_{i}}\right]$ form a system of parameters of $\kappa\left[\frac{X_{0}}{X_{i}}, \cdots, \frac{X_{i-1}}{X_{i}}\right]_{\mathfrak{p}^{\prime}}$, and $t_{i+1}, \cdots, t_{n} \geq 1$.

Let

$$
J_{\mathbb{P}^{n}}^{j}:=\bigoplus_{\mathrm{ht} \mathfrak{p}=j} J(\mathfrak{p})
$$

To define coboundary maps in order to make a residual complex

$$
\cdots \rightarrow 0 \rightarrow J_{\mathbb{P}^{n}}^{0} \rightarrow J_{\mathbb{P}^{n}}^{1} \rightarrow \cdots \rightarrow J_{\mathbb{P}^{n}}^{n} \rightarrow 0 \rightarrow \cdots
$$

on $\mathbb{P}^{n}$, it suffices to define

$$
d_{\mathbb{P} n} ; \mathfrak{p}, \mathfrak{q}: M(\mathfrak{p}) \rightarrow M(\mathfrak{q})
$$

for each pair of points $\mathfrak{p}$ and $\mathfrak{q}$ with ht $\mathfrak{q}=1+$ ht $\mathfrak{p}$. We write $d_{\mathbb{P}^{n} ; \mathfrak{p}, \mathfrak{q}}$ simply as $d_{\mathfrak{p}, \mathfrak{q}}$, if it is clear from the context that we are working on $\mathbb{P}^{n}$. A description of the maps $d_{\mathfrak{p}, \mathfrak{q}}$ can be found in [5]. Here we spell out two special cases of $d_{\mathfrak{p}, \mathfrak{q}}$ that we use is this paper: Let

$$
\omega_{i}^{j_{1} \cdots j_{p}}=d \frac{X_{j_{1}}}{X_{i}} \wedge \cdots \wedge d \frac{X_{j_{p}}}{X_{i}}
$$

and

$$
\omega_{i}=\omega_{i}^{0 \cdots \hat{i} \cdots n}
$$

where $0 \leq i, j_{1}, \cdots, j_{p} \leq n$ and $0 \cdots \hat{i} \cdots n$ is the sequence obtained by removing $i$ from the sequence $0 \cdots n$. The symbols $\omega_{i}^{j_{1} \cdots j_{p}}$ and $\omega_{i}$ are just shorthand for elements in some modules depending on the context.

Formula 1. For each $1 \leq i \leq n$, let $\mathfrak{p}$ (resp. q) be the generic point of $\mathbb{P}^{i}$ (resp. $\left.\mathbb{P}^{i-1}\right)$. The map $d_{\mathfrak{p}, \mathfrak{q}}$ is given by

$$
d_{\mathfrak{p}, \mathfrak{q}}\left(\left[\begin{array}{c}
\left(\frac{X_{i}}{X_{0}}\right)^{-t_{i}} \frac{h}{g} \omega_{0} \\
\left(\frac{X_{i+1}}{X_{0}}\right)^{t_{i+1}}, \cdots,\left(\frac{X_{n}}{X_{0}}\right)^{t_{n}}
\end{array}\right]\right)=\left[\begin{array}{c}
\frac{h}{g} \omega_{0} \\
\left(\frac{X_{i}}{X_{0}}\right)^{t_{i}},\left(\frac{X_{i+1}}{X_{0}}\right)^{t_{i+1}}, \cdots,\left(\frac{X_{n}}{X_{0}}\right)^{t_{n}}
\end{array}\right],
$$

where $t_{i+1}, \cdots, t_{n} \geq 1, t_{i} \in \mathbb{Z}$, and $g, h \in \kappa\left[\frac{X_{1}}{X_{0}}, \cdots, \frac{X_{i}}{X_{0}}\right]$ have no factors $\frac{X_{i}}{X_{0}}$. 
Formula 2. Let $\mathfrak{p}$ and $\mathfrak{q}$ be two points in $\mathbb{P}^{i} \backslash \mathbb{P}^{i-1}$ with ht $\mathfrak{q}=1+$ ht $\mathfrak{p}$ for some $1 \leq i \leq n$. As a prime ideal of $\kappa\left[\frac{X_{0}}{X_{i}}, \cdots, \frac{X_{n}}{X_{i}}\right], \mathfrak{p}$ (resp. q) is generated by a prime ideal $\mathfrak{p}^{\prime}$ (resp. $\left.\mathfrak{q}^{\prime}\right)$ of $\kappa\left[\frac{X_{0}}{X_{i}}, \cdots, \frac{X_{i-1}}{X_{i}}\right]$ and $\frac{X_{i+1}}{X_{i}}, \cdots, \frac{X_{n}}{X_{i}}$. The map $d_{\mathbb{P} n ; \mathfrak{p}, \mathfrak{q}}$ is given by

$$
\begin{aligned}
& d_{\mathbb{P}^{n} ; \mathfrak{p}, \mathfrak{q}}\left(\left[\begin{array}{c}
\frac{h}{g} \omega_{i} \\
f_{1}, \cdots, f_{\ell},\left(\frac{X_{i+1}}{X_{i}}\right)^{t_{i+1}}, \cdots,\left(\frac{X_{n}}{X_{i}}\right)^{t_{n}}
\end{array}\right]\right) \\
& =\left[\begin{array}{c}
\frac{h^{\prime}}{g^{\prime}} \omega_{i} \\
f_{1}^{\prime}, \cdots, f_{\ell+1}^{\prime},\left(\frac{X_{i+1}}{X_{i}}\right)^{t_{i+1}}, \cdots,\left(\frac{X_{n}}{X_{i}}\right)^{t_{n}}
\end{array}\right],
\end{aligned}
$$

where

- $t_{i+1}, \cdots, t_{n} \geq 1$,

- $h, h^{\prime}, f_{1}, \cdots, f_{\ell}, f_{1}^{\prime}, \cdots, f_{\ell+1}^{\prime} \in \kappa\left[\frac{X_{0}}{X_{i}}, \cdots, \frac{X_{i-1}}{X_{i}}\right]$,

- $g \in \kappa\left[\frac{X_{0}}{X_{i}}, \cdots, \frac{X_{i-1}}{X_{i}}\right] \backslash \mathfrak{p}^{\prime}$

- $g^{\prime} \in \kappa\left[\frac{X_{0}}{X_{i}}, \cdots, \frac{X_{i-1}}{X_{i}}\right] \backslash \mathfrak{q}^{\prime}$,

satisfy the following conditions:

- $f_{1}, \cdots, f_{\ell}$ form a system of parameters of $\kappa\left[\frac{X_{0}}{X_{i}}, \cdots, \frac{X_{i-1}}{X_{i}}\right]_{\mathfrak{p}^{\prime}}$,

- $f_{1}^{\prime}, \cdots, f_{\ell+1}^{\prime}$ form a system of parameters of $\kappa\left[\frac{X_{0}}{X_{i}}, \cdots, \frac{X_{i-1}}{X_{i}}\right]_{\mathfrak{q}^{\prime}}$,

$$
d_{\mathbb{P}^{i} ; \mathfrak{p}^{\prime}, \mathfrak{q}^{\prime}}\left(\left[\begin{array}{c}
\frac{h}{g} \omega_{i}^{0 \cdots(i-1)} \\
f_{1}, \cdots, f_{\ell}
\end{array}\right]\right)=\left[\begin{array}{c}
\frac{h^{\prime}}{g^{\prime}} \omega_{i}^{0 \cdots(i-1)} \\
f_{1}^{\prime}, \cdots, f_{\ell+1}^{\prime}
\end{array}\right] .
$$

We remark that it is a subtle problem to give an explicit formula for $h^{\prime}, g^{\prime}$, and $f_{1}^{\prime}, \cdots, f_{\ell+1}^{\prime}$, see [9] Example 3].

The complex $J_{\mathbb{P}^{n}}^{\bullet}$ gives an injective resolution of $\omega_{\mathbb{P}^{n}}:=\Omega_{\mathbb{P}^{n} / \kappa}^{n}$. To describe a short exact sequence

$$
0 \rightarrow \omega_{\mathbb{P}^{n}} \rightarrow J_{\mathbb{P}^{n}}^{0} \rightarrow J_{\mathbb{P}^{n}}^{1}
$$

it suffices to describe a map

$$
\Gamma\left(V_{i}, \omega_{\mathbb{P} n}\right) \rightarrow \Gamma\left(V_{i}, J_{\mathbb{P} n}^{0}\right)
$$

for each $0 \leq i \leq n$. The map we need is trivial in formalism: it has the form

$$
h \omega_{i} \mapsto \frac{h}{1} \omega_{i}
$$

where $h \in \kappa\left[\frac{X_{0}}{X_{i}}, \cdots, \frac{X_{n}}{X_{i}}\right]$.

\section{Complexes Giving Rise to Cohomology}

The residual complex $J_{\mathbb{P} n}^{\bullet}$ can be organized to reveal information about the cohomology modules of locally free sheaves of $\mathbb{P}^{n}$ : Let $\mathbb{P}^{-1}=\emptyset$. For any locally free sheaf $\mathcal{F}$ of $\mathbb{P}^{n}$ and $0 \leq i, j \leq n$, we define

$$
\mathcal{F}^{i, j}:=\bigoplus M(\mathfrak{p}) \otimes \mathcal{O}_{\mathbb{P} n, \mathfrak{p}} \mathcal{F}(n+1)_{\mathfrak{p}},
$$

where $\mathfrak{p}$ ranges over all points in $\mathbb{P}^{i} \backslash \mathbb{P}^{i-1}$ with height $j$. Then $\mathcal{F}^{i, j}=0$ for $j<n-i$, and

$$
\Gamma\left(\mathbb{P}^{n}, J^{j} \otimes \mathcal{F}(n+1)\right) \simeq \bigoplus_{i=n-j}^{n} \mathcal{F}^{i, j}
$$


As $d_{\mathfrak{p}, \mathfrak{q}}$ vanishes unless $\mathfrak{q}$ is an immediate specialization of $\mathfrak{p}$ and the immediate specializations of the points in $\mathbb{P}^{i} \backslash \mathbb{P}^{i-1}$ lies in $\mathbb{P}^{i}$, the non-trivial coboundary maps of the complex $\Gamma\left(\mathbb{P}^{n}, J_{\mathbb{P}^{n}}^{\bullet} \otimes \mathcal{F}(n+1)\right)$ consist of at most those $\mathcal{F}^{i, j} \rightarrow \mathcal{F}^{k, j+1}$ with $i \geq k$. We indicate these maps in the following diagram:

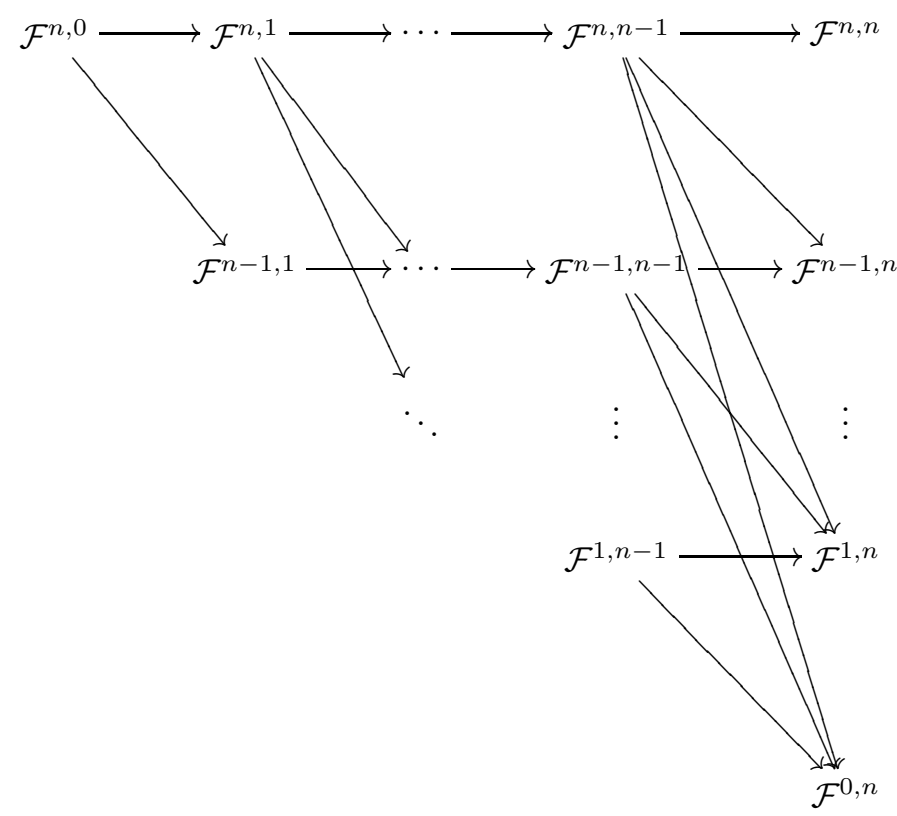

Let $\mathcal{F}^{(n)}=\mathcal{F}^{0, n}$, and let $\mathcal{F}^{(i)}$ be the kernel of the map $\mathcal{F}^{n-i, i} \rightarrow \mathcal{F}^{n-i, i+1}$ for $0 \leq i \leq n-1$.

Lemma 3.1. For each $1 \leq i \leq n$, the subcomplex

$$
\cdots \rightarrow 0 \rightarrow \omega_{\mathbb{P} n}^{n-i, i} \rightarrow \omega_{\mathbb{P} n}^{n-i, i+1} \rightarrow \cdots \rightarrow \omega_{\mathbb{P}^{n}}^{n-i, n} \rightarrow 0 \rightarrow \cdots
$$

of $\Gamma\left(\mathbb{P}^{n}, J_{\mathbb{P}^{n}}^{\bullet}\right)$ has only the $i$-th cohomology non-trivial, which as a $\kappa$-vector space has a basis consisting of the elements of the form

$$
\left[\begin{array}{c}
\left(\frac{X_{0}}{X_{i}}\right)^{t_{0}} \cdots\left(\frac{X_{i-1}}{X_{i}}\right)^{t_{i-1}} \omega_{i} \\
\left(\frac{X_{i+1}}{X_{i}}\right)^{t_{i+1}}, \cdots,\left(\frac{X_{n}}{X_{i}}\right)^{t_{n}}
\end{array}\right],
$$

where $t_{0}, \cdots, t_{i-1} \geq 0$ and $t_{i+1}, \cdots, t_{n} \geq 1$.

Proof. From the construction, $J_{\mathbb{P}^{i}}^{\bullet}$ is a resolution of $\omega_{\mathbb{P}^{i}}$. Thanks to Formula 2 ] the complex $\omega_{\mathbb{P} n}^{n-i, \bullet}$ as a $\kappa$-vector space is a direct sum of $\mathbb{N}^{n-i}$ copies (indexed by $\left(\frac{X_{i+1}}{X_{i}}\right)^{t_{i+1}}, \cdots,\left(\frac{X_{n}}{X_{i}}\right)^{t_{n}}$, where $\left.t_{i+1}, \cdots, t_{n} \geq 1\right)$ of $\Gamma\left(\mathbb{P}^{i} \backslash \mathbb{P}^{i-1}, J_{\mathbb{P}^{i}}^{\bullet}\right.$. Hence $\omega_{\mathbb{P}^{n}}^{n-i, \bullet}$ has only the $(n-i)$-th cohomology non-trivial. Since the kernel of $\omega_{\mathbb{P} i}^{i, 0} \rightarrow \omega_{\mathbb{P} i}^{i, 1}$ consists of elements of the form

$$
h d \frac{X_{0}}{X_{i}} \wedge \cdots \wedge d \frac{X_{i-1}}{X_{i}},
$$

where $h \in \kappa\left[\frac{X_{0}}{X_{i}}, \cdots, \frac{X_{i-1}}{X_{i}}\right]$, the $(n-i)$-th cohomology of $\omega_{\mathbb{P}^{n}}^{n-i, \bullet}$ is generated by the elements of the form

$$
\left[\begin{array}{c}
\left(\frac{X_{0}}{X_{i}}\right)^{t_{0}} \cdots\left(\frac{X_{i-1}}{X_{i}}\right)^{t_{i-1}} \omega_{i} \\
\left(\frac{X_{i+1}}{X_{i}}\right)^{t_{i+1}}, \cdots,\left(\frac{X_{n}}{X_{i}}\right)^{t_{n}}
\end{array}\right]
$$


where $t_{0}, \cdots, t_{i-1} \geq 0$ and $t_{i+1}, \cdots, t_{n} \geq 1$. These elements are linearly independent.

Theorem 3.2. For any locally free sheaf of modules $\mathcal{F}$ on $\mathbb{P}^{n}, \mathrm{H}^{i}\left(\mathbb{P}^{n}, \mathcal{F}\right)$ is isomorphic to the $i$-th cohomology module of the subcomplex

$$
\cdots \rightarrow 0 \rightarrow \mathcal{F}^{(0)} \rightarrow \mathcal{F}^{(1)} \rightarrow \cdots \rightarrow \mathcal{F}^{(n-1)} \rightarrow \mathcal{F}^{(n)} \rightarrow 0 \rightarrow \cdots
$$

of the complex $\Gamma\left(\mathbb{P}^{n}, J_{\mathbb{P}^{n}}^{\bullet} \otimes \mathcal{F}(n+1)\right)$.

Proof. First we compare the complexes $\mathcal{F}^{i, \bullet}$ and $\omega_{\mathbb{P}^{n}}^{i, \bullet}$ for $1 \leq i \leq n$. As modules, $\omega_{\mathbb{P} n}^{i, \bullet}$ is the direct sum of those injective hulls $M(\mathfrak{p})$ with $\mathfrak{p} \in V_{i} \cap \mathbb{P}^{i}$, and $\mathcal{F}^{i, \bullet}$ is the direct sum of rank $\mathcal{F}$ copies of $\omega_{\mathbb{P}^{n}}^{i, \bullet}$. Since the coboundary maps of $\mathcal{F}^{i, \bullet}$ and $\omega_{\mathbb{P}^{n}}^{i, \bullet}$ are given by the coboundary maps of $\Gamma\left(V_{i}, J_{\mathbb{P}^{n}}^{\bullet} \otimes \mathcal{F}(n+1)\right)$ and $\Gamma\left(V_{i}, J_{\mathbb{P}^{n}}^{\bullet}\right)$, respectively, as complexes, $\mathcal{F}^{i} \bullet$ is also isomorphic to the direct sum of $\operatorname{rank} \mathcal{F}$ copies of $\omega_{\mathbb{P}^{n}}^{i, \bullet}$. Therefore $\mathcal{F}^{i, \bullet}$ has only one non-vanishing cohomology $H^{n-i}\left(\mathcal{F}^{i, \bullet}\right)$, which is $\mathcal{F}^{(n-i)}$. With this fact, it is a standard trick to derive the theorem by diagram chasing. The details are left to the reader.

Note that $\mathcal{F}^{(\bullet)}$ is also a subcomplex of

$$
\cdots \rightarrow 0 \rightarrow \mathcal{F}^{n, 0} \rightarrow \mathcal{F}^{n-1,1} \rightarrow \cdots \rightarrow \mathcal{F}^{1, n-1} \rightarrow \mathcal{F}^{0, n} \rightarrow 0 \rightarrow \cdots,
$$

which is isomorphic to the sum of $\operatorname{rank} \mathcal{F}$ copies of

$$
\cdots \rightarrow 0 \rightarrow \omega_{\mathbb{P}^{n}}^{n, 0} \rightarrow \omega_{\mathbb{P}^{n}}^{n-1,1} \rightarrow \cdots \rightarrow \omega_{\mathbb{P}^{n}}^{1, n-1} \rightarrow \omega_{\mathbb{P}^{n}}^{0, n} \rightarrow 0 \rightarrow \cdots
$$

As the coboundary map of the complex (1) is explicitly described in Formula 1 the dimension $h^{i}\left(\mathbb{P}^{n}, \mathcal{F}\right)$ of the $\kappa$-vector space $\mathrm{H}^{i}\left(\mathbb{P}^{n}, \mathcal{F}\right)$ can be calculated directly.

\section{Bott Formula}

The following formula is well-known.

Bott Formula ([1]).

$$
h^{q}\left(\mathbb{P}^{n}, \Omega_{\mathbb{P}^{n} / \kappa}^{p}(m)\right)= \begin{cases}\left(\begin{array}{c}
m-1 \\
p
\end{array}\right)\left(\begin{array}{c}
m+n-p \\
m
\end{array}\right), & \text { for } q=0,0 \leq p \leq n, p<m ; \\
1, & \text { for } m=0,0 \leq p=q \leq n ; \\
\left(\begin{array}{c}
-m-1 \\
n-p
\end{array}\right)\left(\begin{array}{c}
-m+p \\
-m
\end{array}\right), & \text { for } q=n, 0 \leq p \leq n, m<p-n ; \\
0, & \text { otherwise. }\end{cases}
$$

It is interesting to know what the combinatorial numbers that occur in this formula mean. In this section, we interpret these numbers by exhibiting a basis for the $\kappa$-vector space $\mathrm{H}^{q}\left(\mathbb{P}^{n}, \Omega_{\mathbb{P}^{n} / \kappa}^{p}(m)\right)$.

For $i, j_{1}, \cdots, j_{p}, t_{0}, \cdots, t_{n} \in \mathbb{Z}$ satisfying

- $0 \leq i, j_{1}, \cdots, j_{p} \leq n$

- $t_{0}+\cdots+t_{n}=m-p-1$,

we define an element $\omega_{t_{0} \cdots \underline{t}_{i} \cdots t_{n}}^{j_{1} \cdots j_{p}}$ in $\Omega_{\mathbb{P}^{n} / \kappa}^{p}(m)^{n-i, i}$ : If $p>0$,

$$
\omega_{t_{0} \cdots \underline{t}_{i} \cdots t_{n}}^{j_{1} \cdots j_{n}}:=\left[\begin{array}{c}
\left(\frac{X_{0}}{X_{i}}\right)^{t_{0}} \cdots\left(\frac{X_{i-1}}{X_{i}}\right)^{t_{i-1}} \omega_{i} \\
\left(\frac{X_{i+1}}{X_{i}}\right)^{-t_{i+1}}, \cdots,\left(\frac{X_{n}}{X_{i}}\right)^{-t_{n}}
\end{array}\right] \otimes \omega_{i}^{j_{1} \cdots j_{p}} \otimes X_{i}^{m+n+1} .
$$


If $p=0$, the symbol $\omega_{t_{0} \cdots \underline{t_{2}} \cdots t_{n}}^{j_{1} \cdots j_{p}}$ is understood as $\omega_{t_{0} \cdots \underline{t_{i} \cdots t_{n}}}$ and defined by

$$
\omega_{t_{0} \cdots t_{i} \cdots t_{n}}:=\left[\begin{array}{c}
\left(\frac{X_{0}}{X_{i}}\right)^{t_{0}} \cdots\left(\frac{X_{i-1}}{X_{i}}\right)^{t_{i-1}} \omega_{i} \\
\left(\frac{X_{i+1}}{X_{i}}\right)^{-t_{i+1}}, \cdots,\left(\frac{X_{n}}{X_{i}}\right)^{-t_{n}}
\end{array}\right] \otimes X_{i}^{m+n+1} .
$$

These elements satisfy the following properties:

1. $\omega_{t_{0} \cdots t_{i} \cdots t_{n}}^{j_{1} \cdots j_{p}}=0$ if and only if one of the following conditions holds:

- $\overline{j_{\ell}}=i$ for some $\ell$.

- $j_{k}=j_{\ell}$ for some $k \neq \ell$.

- $t_{\ell} \geq 0$ for some $\ell>i$.

2. If $j_{k} \neq j_{\ell}$ for $k \neq \ell$ and $\sigma$ is a permutation of $j_{1} \cdots j_{p}$, then

$$
\omega_{t_{0} \cdots t_{i} \cdots t_{n}}^{j_{1} \cdots j_{p}}=\epsilon(\sigma) \omega_{t_{0} \cdots \underline{t_{i} \cdots t_{n}}}^{\sigma\left(j_{1}\right) \cdots \sigma\left(j_{p}\right)}
$$

where $\epsilon(\sigma)$ is the sign of the permutation $\sigma$.

3. For $0 \leq k \leq n$ not equal $i$,

$$
\frac{X_{k}}{X_{i}} \omega_{t_{0} \cdots t_{i} \cdots t_{n}}^{j_{1} \cdots j_{p}}=\omega_{t_{0}^{\prime} \cdots j_{i}^{\prime} \cdots t_{n}^{\prime}}^{j_{1} \cdots j_{p}}
$$

where $t_{i}^{\prime}=t_{i}-1$ and $t_{\ell}^{\prime}=t_{\ell}+\delta_{k \ell}$ if $\ell \neq i$.

Note that, for $0 \leq i \leq n, \Omega_{\mathbb{P}^{n} / \kappa}^{p}(m)^{(n-i)}$ as a $\kappa$-vector space has a basis consisting of those $\omega_{t_{0} \cdots t_{i} \cdots t_{n}}^{j_{1} \cdots j_{p}}$ satisfying

- $t_{0}, \cdots, \overline{t_{i-1}} \geq 0$

- $t_{i+1}, \cdots, t_{n}<0$,

- $0 \leq j_{1}<\cdots<j_{p} \leq n$,

- $j_{\ell} \neq i$ for all $\ell$.

Given an element

$$
\Psi=\sum a_{t_{0} \cdots t_{n}}^{j_{1} \cdots j_{p}} \omega_{t_{0} \cdots \underline{t_{i}} \cdots t_{n}}^{j_{1} \cdots j_{p}} \quad\left(a_{t_{0} \cdots t_{n}}^{j_{1} \cdots j_{p}} \in \kappa\right)
$$

in $\Omega_{\mathbb{P}^{n} / \kappa}^{p}(m)^{(n-i)}$ satisfying the above conditions, we call $a_{t_{0} \cdots t_{n}}^{j_{1} \cdots j_{p}}$ the coefficient of $\Psi$ at $\omega_{t_{0} \cdots t_{i} \cdots t_{n}}^{j_{1} \cdots j_{p}}$.

Proposition 4.1. For $1 \leq i \leq n$, the map $\Omega_{\mathbb{P}^{n} / \kappa}^{p}(m)^{(n-i)} \rightarrow \Omega_{\mathbb{P}^{n} / \kappa}^{p}(m)^{(n-i+1)}$, denoted by $d^{(n-i)}$, is given by

$$
\omega_{t_{0} \cdots \underline{t_{i}} \cdots t_{n}}^{j_{1} \cdots j_{p}} \mapsto \sum_{\ell=0}^{p}(-1)^{\ell+1} \frac{X_{j_{\ell}}}{X_{i-1}} \omega_{t_{0} \cdots t_{i-1} \cdots t_{n}}^{j_{0} \cdots \hat{j}_{\ell} \cdots j_{p}}
$$

where $j_{0}=i$.

Proof. Assume first that $p=0$. Write $\omega_{t_{0} \cdots t_{i} \cdots t_{n}}$ in terms of $\frac{X_{1}}{X_{0}}, \cdots, \frac{X_{n}}{X_{0}}$ and $X_{0}$ :

$$
\omega_{t_{0} \cdots t_{i} \cdots t_{n}}=(-1)^{i}\left[\begin{array}{c}
\left(\frac{X_{1}}{X_{0}}\right)^{t_{1}} \cdots\left(\frac{X_{i-1}}{X_{0}}\right)^{t_{i-1}}\left(\frac{X_{i}}{X_{0}}\right)^{t_{i}+1} \omega_{0} \\
\left(\frac{X_{i+1}}{X_{0}}\right)^{-t_{i+1}}, \cdots,\left(\frac{X_{n}}{X_{0}}\right)^{-t_{n}}
\end{array}\right] \otimes X_{0}^{m+n+1}
$$

By Formula 1, $\omega_{t_{0} \cdots \underline{t_{i}} \cdots t_{n}}$ maps to

$$
(-1)^{i}\left[\begin{array}{c}
\left(\frac{X_{1}}{X_{0}}\right)^{t_{1}} \cdots\left(\frac{X_{i-1}}{X_{0}}\right)^{t_{i-1}} \omega_{0} \\
\left(\frac{X_{i}}{X_{0}}\right)^{-t_{i}-1},\left(\frac{X_{i+1}}{X_{0}}\right)^{-t_{i+1}}, \cdots,\left(\frac{X_{n}}{X_{0}}\right)^{-t_{n}}
\end{array}\right] \otimes X_{0}^{m+n+1},
$$

which equals $-\frac{X_{i}}{X_{i-1}} \omega_{t_{0} \cdots \underline{t_{i-1}} \cdots t_{n}}$. 
Now assume $p>0$. If we switch $j_{k}$ and $j_{k+1}$ for some $1 \leq k<p$, then it is

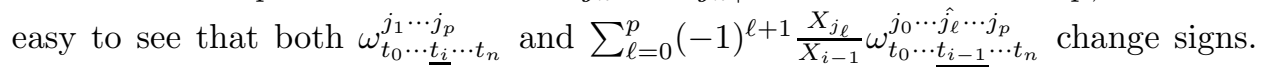
If $j_{k}=j_{k+1}$ for some $1 \leq \bar{k}<p$, then it is easy to see that both $\omega_{t_{0} \cdots \underline{t}_{i} \cdots t_{n}}^{j_{1} \cdots j_{p}}$ and $\sum_{\ell=0}^{p}(-1)^{\ell+1} \frac{X_{j_{\ell}}}{X_{i-1}} \omega_{t_{0} \cdots \underline{t_{i-1}} \cdots t_{n}}^{j_{0} \cdots \hat{j}_{p}}$ vanish. Therefore we may assume that $\overline{0} \leq j_{1}<$ $\cdots<j_{p} \leq n$. If $j_{k}=i$ for some $1 \leq k \leq p$, it is also easy to see that both $\omega_{t_{0} \cdots t_{i} \cdots t_{n}}^{j_{1} \cdots j_{p}}$ and $\sum_{\ell=0}^{p}(-1)^{\ell+1} \frac{X_{j_{\ell}}}{X_{i-1}} \omega_{t_{0} \cdots t_{i-1} \cdots t_{n}}^{j_{0} \cdots \hat{j_{\ell}} \cdots j_{p}}$ vanish. Therefore we may assume furthermore that $j_{k} \neq i$ for $1 \leq k \leq p$.

Write $\omega_{t_{0} \cdots \underline{t}_{i} \cdots t_{n}}^{j_{1} \cdots j_{p}}$ in terms of $\frac{X_{1}}{X_{0}}, \cdots, \frac{X_{n}}{X_{0}}$ and $X_{0}$ :

$\omega_{t_{0} \cdots \underline{t_{i} \cdots t_{n}}}^{j_{1} \cdots j_{p}}=\left[\begin{array}{c}\left(\frac{X_{1}}{X_{0}}\right)^{t_{1}} \cdots\left(\frac{X_{i}}{X_{0}}\right)^{t_{i}} \omega_{0} \\ \left(\frac{X_{i+1}}{X_{0}}\right)^{-t_{i+1}}, \cdots,\left(\frac{X_{n}}{X_{0}}\right)^{-t_{n}}\end{array}\right] \otimes \sum_{\ell=0}^{p}(-1)^{i+\ell} \frac{X_{j_{\ell}}}{X_{0}} \omega_{0}^{j_{0} \cdots \hat{j}_{\ell} \cdots j_{p}} \otimes X_{0}^{m+n+1}$

By Formula 1, $\omega_{t_{0} \cdots \underline{t}_{i} \cdots t_{n}}^{j_{1} \cdots j_{p}}$ maps to

$$
\left[\begin{array}{c}
\left(\frac{X_{1}}{X_{0}}\right)^{t_{1}} \cdots\left(\frac{X_{i-1}}{X_{0}}\right)^{t_{i-1}} \omega_{0} \\
\left(\frac{X_{i}}{X_{0}}\right)^{-t_{i}}, \cdots,\left(\frac{X_{n}}{X_{0}}\right)^{-t_{n}}
\end{array}\right] \otimes \sum_{\ell=0}^{p}(-1)^{i+\ell} \frac{X_{j_{\ell}}}{X_{0}} \omega_{0}^{j_{0} \cdots \hat{j}_{\ell} \cdots j_{p}} \otimes X_{0}^{m+n+1}
$$

which equals

$$
\begin{aligned}
& {\left[\begin{array}{c}
\left(\frac{X_{0}}{X_{i-1}}\right)^{t_{0}} \cdots\left(\frac{X_{i-2}}{X_{i-1}}\right)^{t_{i-2}} \omega_{i-1} \\
\left(\frac{X_{i}}{X_{i-1}}\right)^{-t_{i}}, \cdots,\left(\frac{X_{n}}{X_{i-1}}\right)^{-t_{n}}
\end{array}\right] \otimes \sum_{\ell=0}^{p}(-1)^{\ell+1} \frac{X_{j_{\ell}}}{X_{0}}} \\
& \cdot\left(\frac{X_{0}}{X_{i-1}} \omega_{i-1}^{j_{0} \cdots \hat{j}_{\ell} \cdots j_{p}}-\frac{X_{j_{0}}}{X_{i-1}} \omega_{i-1}^{0 j_{1} \cdots \hat{j}_{\ell} \cdots j_{p}}+\frac{X_{j_{1}}}{X_{i-1}} \omega_{i-1}^{0 j_{0} j_{2} \cdots \hat{j}_{\ell} \cdots j_{p}}-\cdots\right) \otimes X_{i-1}^{m+n+1} .
\end{aligned}
$$

If $j_{1}>0$, the element $\omega_{i-1}^{0 j_{0} \cdots \hat{j_{k}} \cdots \hat{j_{\ell}} \cdots j_{p}}$ appears twice in the above summation with opposite signs for each $0 \leq k<\ell \leq p$. If $j_{1}=0$, the above summation has only one non-trivial term, namely the term $\ell=1$. In either cases, the above element equals

$$
\sum_{\ell=0}^{p}(-1)^{\ell+1} \frac{X_{j_{\ell}}}{X_{i-1}} \omega_{t_{0} \cdots \underline{t_{i-1}} \cdots t_{n}}^{j_{0} \cdots \hat{j}_{p}}
$$

Definition 4.2. For $0 \leq i \leq n-p, V_{p, m}^{i}$ is defined to be the vector space generated by those

$$
\sum_{\ell=0}^{p}(-1)^{\ell+1} \frac{X_{j_{\ell}}}{X_{n}} \omega_{t_{0} \cdots t_{n-i} 0 \cdots \underline{0}}^{j_{0} \cdots \hat{j_{\ell} \cdots j_{p}}}
$$

satisfying the conditions

- $t_{0}, \cdots, t_{n-i} \geq 0$

- $j_{0}<\cdots<j_{p}=n-i$.

\section{Theorem 4.3.}

$$
\operatorname{Kernel}\left(d^{(0)}\right)=V_{p, m}^{0} \oplus \cdots \oplus V_{p, m}^{n-p}
$$


Proof. If $p=0$, then

$$
\begin{aligned}
\operatorname{Kernel}\left(d^{(0)}\right) & \left.=\left\langle\omega_{t_{0} \cdots \underline{t_{n}}}\right| t_{0}, \cdots, t_{n-1} \geq 0 \text { and } t_{n} \geq-1\right\rangle, \\
V_{p, m}^{0} & =\left\langle\omega_{t_{0} \cdots \underline{t_{n}}} \mid t_{0}, \cdots, t_{n} \geq 0\right\rangle, \\
V_{p, m}^{n} & =\left\langle\omega_{m 0 \cdots 0 \underline{(-1)}}\right\rangle \\
V_{p, m}^{i} & \left.=\left\langle\omega_{t_{0} \cdots t_{n-i} 0 \cdots 0 \underline{(-1)}}\right| t_{0}, \cdots, t_{n-i-1} \geq 0 \text { and } t_{n-i} \geq 1\right\rangle
\end{aligned}
$$

for $0<i<n$. It is easy to see (2) in such case.

Now we assume that $p>0$. It is straightforward to check that the vector spaces $V_{p, m}^{0}, \cdots, V_{p, m}^{n-p}$ are contained in the kernel of the map $d^{(0)}$. For $-1 \leq i \leq n-p$, we define

$$
W_{n-i}:=\operatorname{Kernel}\left(d^{(0)}\right) \cap\left\langle\omega_{t_{0} \cdots t_{n-i-1} 0 \cdots 0 \underline{(-1)}}^{j_{1} \cdots j_{p}} \mid j_{1}<\cdots<j_{p}<n-i\right\rangle .
$$

Note that $W_{n+1}=\operatorname{Kernel}\left(d^{(0)}\right)$ and $W_{p}=0$. We will show that

$$
W_{n-i+1}=W_{n-i} \oplus V_{p, m}^{i}
$$

for $0 \leq i \leq n-p$, from which the theorem follows easily.

We first show that $W_{n-i+1}=W_{n-i}+V_{p, m}^{i}$. Let

$$
\Psi=\sum a_{t_{0} \cdots t_{n-i}}^{j_{1} \cdots j_{p}} \omega_{t_{0} \cdots t_{n-i} 0 \cdots 0 \underline{(-1)}}^{j_{1} \cdots j_{p}} \quad\left(a_{t_{0} \cdots t_{n-i}}^{j_{1} \cdots j_{p}} \in \kappa\right)
$$

be an element in $W_{n-i+1}$, where the summation runs over those $t_{0}, \cdots, t_{n-i}$ and $j_{1}, \cdots, j_{p}$ satisfying the conditions

- $t_{0}, \cdots, t_{n-i} \geq 0$,

- $j_{1}<\cdots<j_{p}<n-i+1$.

By subtracting from $\Psi$ the element

$$
\begin{aligned}
\sum a_{t_{0} \cdots t_{n-i-1}\left(t_{n-i}+1\right)}^{j_{1} \cdots j_{p}} & \left(\omega_{t_{0} \cdots t_{n-i-1}\left(t_{n-i}+1\right) 0 \cdots 0}^{j_{1} \cdots j_{p}} \underline{(-1)}\right. \\
& \left.+\sum_{\ell=1}^{p}(-1)^{\ell+p+1} \frac{X_{j_{\ell}}}{X_{n}} \omega_{t_{0} \cdots t_{n-i} \cdots \underline{j_{\ell} \cdots j_{p}(n-i)}}^{j_{1} \cdots \hat{t}_{0}}\right)
\end{aligned}
$$

in $V_{p, m}^{i}$, where the summation runs over those $t_{0}, \cdots, t_{n-i}, j_{1}, \cdots, j_{p}$ satisfying the conditions

- $t_{0}, \cdots, t_{n-i} \geq 0$,

- $j_{1}<\cdots<j_{p}<n-i$,

we may assume that the representation (3) of $\Psi$ satisfies the additional condition

- $j_{p}<n-i, t_{n-i}=0$

or the additional condition

- $j_{p}=n-i$.

In other words, we may assume that

$$
\Psi=\Psi_{1}+\Psi_{2},
$$

where

$$
\Psi_{1}=\sum a_{t_{0} \cdots t_{n-i-1} 0}^{j_{1} \cdots j_{p}} \omega_{t_{0} \cdots t_{n-i-1} 0 \cdots 0 \underline{(-1)}}^{j_{1} \cdots j_{p}}
$$

with indices running over those $t_{0}, \cdots, t_{n-i-1}, j_{1}, \cdots, j_{p}$ satisfying the conditions

- $t_{0}, \cdots, t_{n-i-1} \geq 0$, 
- $j_{1}<\cdots<j_{p}<n-i$;

and

$$
\Psi_{2}=\sum a_{t_{0} \cdots t_{n-i}}^{j_{1} \cdots j_{p-1}(n-i)} \omega_{t_{0} \cdots t_{n-i} 0 \cdots 0 \underline{(-1)}}^{j_{1} \cdots j_{p-1}(n-i)}
$$

with indices running over those $t_{0}, \cdots, t_{n-i}, j_{1}, \cdots, j_{p-1}$ satisfying the conditions

- $t_{0}, \cdots, t_{n-i} \geq 0$

- $j_{1}<\cdots<j_{p-1}<n-i$.

The coefficient of $d^{(0)}(\Psi)$ at $\omega_{t_{0} \cdots t_{n-i-1}\left(t_{n-i}+1\right) 0 \cdots 0 \underline{(-1)(-1)}}^{n j_{1} \cdots j_{p-1}}$ is

$$
(-1)^{p+1} a_{t_{0} \cdots t_{n-i}}^{j_{1} \cdots j_{p-1}(n-i)},
$$

which has to be zero. Hence $\Psi_{2}=0$, and it follows that $W_{n-i+1}=W_{n-i}+V_{p, m}^{i}$.

Now we show that $W_{n-i} \cap V_{p, m}^{i}=0$. For $i=0$, this is trivial. So we assume that $i>0$. Let

$$
\Psi=\sum b_{t_{0} \cdots t_{n-i}}^{j_{0} \cdots j_{p}}\left(\sum_{\ell=0}^{p}(-1)^{\ell+1} \frac{X_{j_{\ell}}}{X_{n}} \omega_{t_{0} \cdots t_{n-i} 0 \cdots \underline{0}}^{j_{0} \cdots \hat{j}_{\ell} \cdots j_{p}}\right)
$$

be an element in $W_{n-i} \cap V_{p, m}^{i}$, where the (first) summation runs over those $t_{0}, \cdots$, $t_{n-i}$ and $j_{0}, \cdots, j_{p}$ satisfying the conditions

- $t_{0}, \cdots, t_{n-i} \geq 0$

- $j_{0}<\cdots<j_{p}=n-i$.

As an element in $V_{p, m}^{i}$, the coefficient of $\Psi$ at $\omega_{t_{0} \cdots t_{n-i-1}\left(t_{n-i}+1\right) 0 \cdots 0 \underline{(-1)}}^{j_{0} \cdots j_{p-1}}$ is

$$
(-1)^{p+1} b_{t_{0} \cdots t_{n-i}}^{j_{0} \cdots j_{p}}
$$

However, as an element in $W_{n-i}$, the coefficient of $\Psi$ at $\omega_{t_{0} \cdots t_{n-i-1}\left(t_{n-i}+1\right) 0 \cdots 0(-1)}^{j_{0} \cdots j_{p-1}}$ is zero. Therefore all $b_{t_{0} \cdots t_{n-i}}^{j_{0} \cdots j_{p}}$ vanish, and hence $\Psi=0$.

\section{Corollary 4.4.}

$$
h^{0}\left(\mathbb{P}^{n}, \Omega_{\mathbb{P}^{n} / \kappa}^{p}(m)\right)=\left(\begin{array}{c}
m-1 \\
p
\end{array}\right)\left(\begin{array}{c}
m+n-p \\
m
\end{array}\right) .
$$

Proof. Modulo the subspace generated by the elements of the form $\omega_{t_{0}^{\prime} \cdots j_{n}^{\prime}}^{j_{1}^{\prime} \cdots j^{\prime}}(n-i)$, the elements

$$
\sum_{\ell=0}^{p}(-1)^{\ell+1} \frac{X_{j_{\ell}}}{X_{n}} \omega_{t_{0} \cdots t_{n-i} 0 \cdots \underline{0}}^{j_{0} \cdots \hat{j}_{\ell} \cdots j_{p}} \text { and } \quad(-1)^{p+1} \omega_{t_{0} \cdots t_{n-i-1}\left(t_{n-i}+1\right) 0 \cdots 0}^{j_{0} \cdots j_{p-1}}
$$

$\left(j_{0}<\cdots<j_{p}=n-i\right)$ are the same. Hence, for a fixed $i$, the elements

$$
\sum_{\ell=0}^{p}(-1)^{\ell+1} \frac{X_{j_{\ell}}}{X_{n}} \omega_{t_{0} \cdots t_{n-i} 0 \cdots \underline{0}}^{j_{0} \cdots \hat{j}_{\ell} \cdots j_{p}}
$$


are linearly independent. Therefore

$$
\begin{aligned}
h^{0}\left(\mathbb{P}^{n}, \Omega_{\mathbb{P}^{n} / \kappa}^{p}(m)\right) & =\sum_{i=0}^{n-p} \operatorname{dim} V_{p, m}^{i} \\
& =\sum_{i=0}^{n-p}\left(\begin{array}{c}
n-i \\
p
\end{array}\right)\left(\begin{array}{c}
m+n-p-i-1 \\
n-i
\end{array}\right) \\
& =\left(\begin{array}{c}
m-1 \\
p
\end{array}\right) \sum_{i=0}^{n-p}\left(\begin{array}{c}
m+n-p-i-1 \\
m-1
\end{array}\right) \\
& =\left(\begin{array}{c}
m-1 \\
p
\end{array}\right)\left(\begin{array}{c}
m+n-p \\
m
\end{array}\right) .
\end{aligned}
$$

Theorem 4.5. For $0<q<n$, $\mathrm{H}^{q}\left(\mathbb{P}^{n}, \Omega_{\mathbb{P}^{n} / \kappa}^{p}(m)\right)$ vanishes unless $m=0$ and $p=q ; \mathrm{H}^{q}\left(\mathbb{P}^{n}, \Omega_{\mathbb{P}^{n} / \kappa}^{q}\right)$ is non-trivial and generated (after identifying with the $q$-th cohomology of $\left.\Omega_{\mathbb{P}^{n} / \kappa}^{q(\bullet)}\right)$ by the equivalence class containing $\omega_{0 \cdots 0(-1) \cdots(-1)}^{(n-q+1)(n-q+2) \cdots n}$.

Proof. If $p=0$, then

$$
\operatorname{Kernel}\left(d^{(q)}\right)=\left\langle\omega_{t_{0} \cdots t_{n-q} \cdots t_{n}} \mid t_{n-q} \geq-1\right\rangle=\operatorname{Image}\left(d^{(q-1)}\right) .
$$

This implies $\mathrm{H}^{q}\left(\mathbb{P}^{n}, \mathcal{O}_{\mathbb{P}^{n}}(m)\right)=0$. So, to prove the theorem, we may assume that $p>0$.

Let $\Psi$ be an element in the kernel of the map

$$
d^{(q)}: \Omega_{\mathbb{P}^{n} / \kappa}^{p}(m)^{(q)} \rightarrow \Omega_{\mathbb{P}^{n} / \kappa}^{p}(m)^{(q+1)} .
$$

We claim that, by adding a suitable element in the image of $d^{(q-1)}, \Psi$ becomes

$$
\begin{cases}\omega_{0 \cdots 0(-1) \cdots(-1)}^{(n-q+1)(n-q+2) \cdots n}, & \text { if } m=0 \text { and } p=q \\ 0, & \text { otherwise. }\end{cases}
$$

Recall that $\Psi$ can be written as

$$
\Psi=\sum a_{t_{0} \cdots t_{n}}^{j_{1} \cdots j_{p}} \omega_{t_{0} \cdots t_{n-q} \cdots t_{n}}^{j_{1} \cdots j_{p}}
$$

where $a_{t_{0} \cdots t_{n}}^{j_{1} \cdots j_{p}} \in \kappa$ and the summation runs over those $t_{0}, \cdots, t_{n}, j_{1}, \cdots, j_{q} \in \mathbb{Z}$ satisfying

- $t_{0}, \cdots, t_{n-q-1} \geq 0$

- $t_{n-q+1}, \cdots, t_{n}<0$

- $t_{0}+\cdots+t_{n}=m-p-1$,

- $0 \leq j_{1}<\cdots<j_{p} \leq n$,

- $j_{\ell} \neq n-q$ for all $\ell$.

Step 1. We may assume $t_{n-q}=-1$.

If $t_{n-q} \leq-2$ and $j_{\ell} \neq n-q-1$ for all $\ell$, then the coefficient of $d^{(q)}(\Psi)$ at $\frac{X_{n-q}}{X_{n-q-1}} \omega_{t_{0} \cdots t_{n-q-1} \cdots t_{n}}^{j_{1} \cdots j_{p}}$ is $-a_{t_{0} \cdots t_{n}}^{j_{1} \cdots j_{p}}$, which has to be zero. Using this fact, one sees that if $t_{n-q} \leq-2$ and $j_{\ell}=n-q-1$ for some $\ell$, then the coefficient of $d^{(q)}(\Psi)$ at $\omega_{t_{0} \cdots t_{n-q}-1}^{(n-q) j_{1} \cdots \hat{j}_{\ell} \cdots j_{p}}$ is $(-1)^{\ell+1} a_{t_{0} \cdots t_{n}}^{j_{1} \cdots j_{p}}$, which is thus also zero. Therefore $a_{t_{0} \cdots t_{n}}^{j_{1} \cdots j_{p}}=0$ for $t_{n-q} \frac{t_{n-q-1}}{\leq-2}$. 
For $t_{n-q} \geq 0$, if $j_{\ell} \neq n-q+1$ for all $\ell$, then

$$
\omega_{t_{0} \cdots t_{n-q} \cdots t_{n}}^{j_{1} \cdots j_{p}}+d^{(q-1)}\left(\frac{X_{n-q}}{X_{n-q+1}} \omega_{t_{0} \cdots t_{n-q+1} \cdots t_{n}}^{j_{1} \cdots j_{p}}\right)
$$

is contained in the $\kappa$-vector space generated by those $\omega_{t_{0}^{\prime} \cdots t_{n-q}^{\prime} \cdots t_{n}^{\prime}}^{(n-q+1) j_{p}^{\prime} \cdots j_{p}^{\prime}}$ satisfying $t_{n-q}^{\prime} \geq 0$. So we may assume that $j_{\ell}=n-q+1$ for some $\ell$. In this case,

$$
\omega_{t_{0} \cdots \underline{t_{n-q} \cdots t_{n}}}^{j_{1} \cdots j_{p}}=d^{(q-1)}\left((-1)^{\ell-1} \omega_{t_{0} \cdots \underline{t_{n-q+1} \cdots t_{n}}}^{(n-q) j_{1} \cdots \hat{j}_{j} \cdots j_{p}}\right) .
$$

Therefore, to prove the claim, we may assume that the representation (4) of $\Psi$ satisfies the additional condition:

- $t_{n-q}=-1$.

Step 2. We may assume $j_{\ell_{0}}=n-q+1$ for some $\ell_{0}$ and $t_{n-q+1}=-1$.

If $j_{\ell} \neq n-q+1$ for all $\ell$, then

$$
\omega_{t_{0} \cdots t_{n-q-1}}^{j_{1} \cdots j_{p}} \underline{\underline{(-1)} t_{n-q+1} \cdots t_{n}}+d^{(q-1)}\left(\omega_{t_{0} \cdots t_{n-q-1} 0 \underline{\left(t_{n-q+1}-1\right)} t_{n-q+2} \cdots t_{n}}^{j_{1} \cdots j_{p}}\right)
$$

is contained in the $\kappa$-vector space generated by those $\omega_{t_{0}^{\prime} \cdots t_{n-q-1}^{\prime}}^{(n-1) j_{2}^{\prime} \cdots j_{p}^{\prime}} t_{n-q+1}^{\prime} \cdots t_{n}^{\prime}$. Therefore, by adding an element of the image of $d^{(q-1)}$ to $\Psi$, we may assume furthermore that the representation (4) of $\Psi$ satisfies one more condition:

- $j_{\ell_{0}}=n-q+1$ for some $\ell_{0}$.

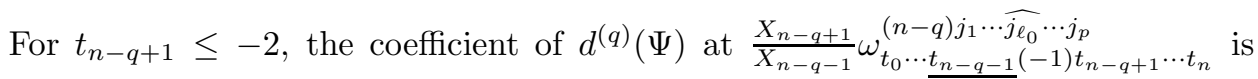
$(-1)^{\ell_{0}+1} a_{t_{0} \cdots t_{n-q-1}(-1) t_{n-q+1} \cdots t_{n}}^{j_{1} \cdots j_{p}}$, which has to be zero. Therefore the representation (4) of $\Psi$ also satisfies the condition

- $t_{n-q+1}=-1$.

Step 3. We may assume $q \leq p, t_{n-q}=\cdots=t_{n}=-1$, and $j_{p-q+i}=n-q+i$ for $1 \leq i \leq q$.

If $q>p$, repeating the process in Step [2] we may assume that the representation (44) is of the form

$$
\Phi=\sum a_{t_{0} \cdots t_{n-q-1}(-1) \cdots(-1) t_{n-q+p+1} \cdots t_{n}}^{(n-q+1) \cdots(n-q+p)} \omega_{t_{0} \cdots t_{n-q-1}}^{(n-q+1) \cdots(n-q+p)} \underline{(-1) \cdots(-1) t_{n-q+p+1} \cdots t_{n}} .
$$

Since

$$
\begin{gathered}
d^{(q-1)}\left(\omega_{t_{0} \cdots t_{n-q-1}}^{(n-q+2) \cdots(n-q+p+1)} \underline{(-1) \cdots(-1)\left(t_{n-q+p+1}-1\right) t_{n-q+p+2} \cdots t_{n}}\right) \\
=(-1)^{p+1} \omega_{t_{0} \cdots t_{n-q-1}}^{(n-q)+1) \cdots(n-q+p)} \underline{(-1) t_{n-q+p+1} \cdots t_{n}},
\end{gathered}
$$

by adding to $\Psi$ an element of the image of $d^{(q-1)}, \Psi$ becomes zero.

For $q \leq p$, repeating the process in Step 2 we may write

$$
\Phi=\sum a_{t_{0} \cdots t_{n-q-1}(-1) \cdots(-1)}^{j_{1} \cdots j_{p-q}(n-q+1) \cdots n} \omega_{t_{0} \cdots t_{n-q-1}}^{j_{1} \cdots j_{p-q}(n-1) \cdots(-1)} .
$$

Step 4. We may assume $q=p$.

Assume $p>q$. If $t_{0}>0$ and $j_{1}>0$, then

$$
\omega_{t_{0} \cdots t_{n-q-1}}^{j_{1} \cdots j_{p-q}(n-q+1)(n-q+2) \cdots n}-d^{(q-1)}\left((-1)^{p-q} \omega_{\left(t_{0}-1\right) t_{1} \cdots t_{n-q-1} 0 \underline{(-1) \cdots(-1)}}^{0 j_{1} \cdots j_{p-q}(n-q+2)(n-q+3) \cdots n}\right)
$$




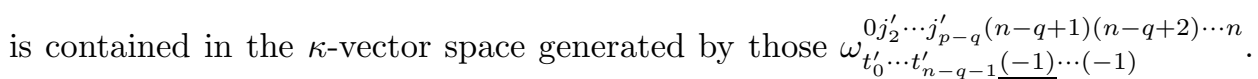
Therefore, in the case $p>q$, we may assume that the representation (15) also satisfies the condition

- $t_{0}=0$ or $j_{1}=0$.

If $j_{1}=0$, the coefficient of $d^{(q)}(\Psi)$ at $\omega_{\left(t_{0}+1\right) t_{1} \cdots t_{n-q-2}\left(t_{n-q-1}-1\right)(-1) \cdots(-1)}^{(n-q) j_{2} \cdots j_{p-q}(n-q+1)(n-q+2) \cdots n}$ is

$$
a_{t_{0} \cdots t_{n-q-1}(-1) \cdots(-1)}^{0 j_{2} \cdots j_{p-q}(n-q+1)(n-q+2) \cdots n}
$$

which has to be zero. Therefore we may assume that the representation (5) satisfies the condition

- $t_{0}=0$ and $j_{1}>0$.

Repeating this process, finally we may assume that $\Psi$ is of the form

$$
\Phi=\sum a_{0 \cdots 0 t_{n-p} \cdots t_{n-q-1}(-1) \cdots(-1)}^{(n-p) \cdots(n-q-1)(n-q+1) \cdots n} \omega_{0 \cdots 0 t_{n-p} \cdots t_{n-q-1}}^{(n-1) \cdots(-1)}
$$

For such a representation, the coefficient of $d^{(q)}(\Psi)$ at $\omega_{0 \cdots 0 t_{n-p} \cdots t_{n-q-1}}^{(n-p) \cdots(n-q) \cdots(-1)}(n-q) \cdots n$ is $a_{0 \cdots 0 t_{n-p} \cdots t_{n-q-1}(-1) \cdots(-1)}^{(n-p) \cdots(n-q-1)(n-q+1) \cdots n}$, which has to be zero. Therefore, if $p>q$, adding to $\Psi$ a suitable element in the image of $d^{(q-1)}, \Psi$ becomes zero. After these steps, we may write

$$
\Phi=\sum a_{t_{0} \cdots t_{n-q-1}(-1) \cdots(-1)}^{(n-q+1) \cdots n} \omega_{t_{0} \cdots t_{n-q-1} \underline{(-1) \cdots(-1)}}^{(n-q+1) \cdots n} .
$$

Step 5. We may assume $m=0$ and $t_{0}=\cdots=t_{n-q-1}=0$.

If $m<0$, it is impossible that the conditions

- $t_{0}, \cdots, t_{n-q-1} \geq 0$

- $t_{0}+\cdots+t_{n-q-1}-q-1=m-q-1$

of the representation (6) are satisfied. Hence $\Psi=0$ if $m<0$. If $m>0$, that is, $t_{\ell}>0$ for some $0 \leq \ell \leq n-q-1$, then

$$
d^{(q-1)}\left(\omega_{t_{0} \cdots t_{\ell-1}\left(t_{\ell}-1\right) t_{\ell+1} \cdots t_{n-q-1}}^{\ell(n-q) \cdots(-1)}\right)=\omega_{t_{0} \cdots t_{n-q-1} \underline{(-1)}(n-q)(-1)}^{(n-q+2) \cdots n} .
$$

Hence we may assume that the representation (6) of $\Psi$ satisfies the conditions

- $m=0$,

- $t_{0}=\cdots=t_{n-q-1}=0$,

that is, we may assume that $\Psi$ is generated by $\omega_{0 \cdots 0}^{(n-q+1)(n-q+2) \cdots n}$. This final step verifies the claim.

It is easy to check that $\omega_{0 \cdots 0(-1)}^{(n-q+1)(n-q+2) \cdots n}$ is in the kernel of $d^{(q)}$. To prove the theorem, it remains to show that $\omega_{0 \cdots 0(-1)(-1) \cdots(-1)}^{(n-q+1)(n-q+2) \cdots n}$ is not in the image of $d^{(q-1)}$.

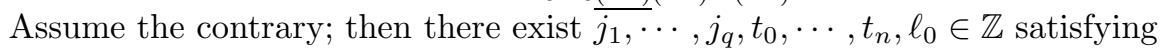

- $t_{0}, \cdots, t_{n-q} \geq 0$,

- $t_{n-q+2}, \cdots, t_{n}<0$,

- $t_{0}+\cdots+t_{n}=-q-1$,

- $0 \leq j_{1}<\cdots<j_{q} \leq n$,

- $j_{\ell} \neq n-q+1$ for all $\ell$

such that

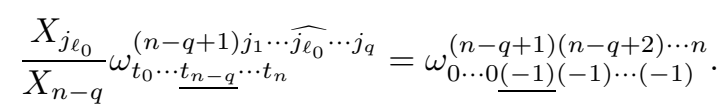


$\left\{n-q+1, j_{1}, \cdots, \widehat{j_{\ell_{0}}}, \cdots, j_{q}\right\}=\{n-q+1, \cdots, n\}$ implies that $j_{\ell_{0}} \leq n-q$. This contradicts $t_{j_{\ell_{0}}} \geq 0$.

Corollary 4.6. For $0<q<n$,

$$
h^{q}\left(\mathbb{P}^{n}, \Omega_{\mathbb{P}^{n} / \kappa}^{p}(m)\right)= \begin{cases}1, & \text { if } m=0 \text { and } p=q \\ 0, & \text { otherwise. }\end{cases}
$$

Definition 4.7. For $0 \leq i \leq p, \check{V}_{p, m}^{n-i}$ is defined to be the vector space generated by those $\omega_{(-1) \cdots(-1) t_{i} \cdots t_{n}}^{12 \cdots i j_{i+1} \cdots j_{p}}$ satisfying

- $i<j_{i+1}<\cdots<j_{p} \leq n$,

- $t_{i}<-1$,

- $t_{i+1}, \cdots, t_{n}<0$,

- $t_{i}+\cdots+t_{n}=m-p+i-1$.

Note that the elements $\omega_{(-1) \cdots(-1) t_{i} \cdots t_{n}}^{12 \cdots i j_{i+1} \cdots j_{p}}$ satisfying the above conditions are linearly independent.

\section{Theorem 4.8.}

$$
\Omega_{\mathbb{P}^{n} / \kappa}^{p}(m)^{(n)}=\check{V}_{p, m}^{n-p} \oplus \cdots \oplus \check{V}_{p, m}^{n} \oplus \operatorname{Image}\left(d^{(n-1)}\right)
$$

Proof. If $p=0$, then

$$
\operatorname{Image}\left(d^{(n-1)}\right)=\left\langle d^{(n-1)}\left(\omega_{t_{0}} \underline{t_{1} \cdots t_{n}}\right) \mid t_{0} \geq 0\right\rangle=\left\langle\omega_{\underline{t_{0}} \cdots t_{n}} \mid t_{0} \geq-1\right\rangle .
$$

As

$$
\check{V}_{p, m}^{n}=\left\langle\omega_{\underline{t_{0}} \cdots t_{n}} \mid t_{0}<-1\right\rangle,
$$

it is easy to verify the theorem in this case. If $p=n$, then

$$
\begin{aligned}
\operatorname{Image}\left(d^{(n-1)}\right) & =\left\langle\omega_{\underline{t_{0} \cdots t_{n}}}^{1 \cdots n} \mid t_{0} \geq 0\right\rangle \\
\check{V}_{p, m}^{n-i} & =\left\langle\omega_{\underline{(-1)} \cdots(-1) t_{i} \cdots t_{n}}^{1 \cdots n} \mid t_{i}<-1\right\rangle
\end{aligned}
$$

for $0 \leq i \leq n$. It is also easy to verify the theorem in this case. So we assume that $0<p<n$ in the rest of the proof.

In the proof, we assume that the indices $j_{1}, \cdots, j_{p}$ satisfy the condition $j_{1}<$ $\cdots<j_{p}$. We define

$$
\begin{aligned}
W_{0}^{\prime} & :=\left\langle d^{(n-1)}\left(\omega_{t_{0} \underline{t_{1}} t_{2} \cdots t_{n}}^{j_{1} \cdots j_{2}}\right) \mid j_{1}=0\right\rangle, \\
W_{0}^{\prime \prime} & \left.:=\left\langle d^{(n-1)}\left(\omega_{t_{0} \underline{t_{1}} t_{2} \cdots t_{n}}^{j_{1} j_{p}}\right)\right| j_{1}>0 \text { and } t_{0}>0\right\rangle, \\
W_{0} & :=W_{0}^{\prime}+W_{0}^{\prime \prime}, \\
W_{1} & \left.:=\left\langle d^{(n-1)}\left(\omega_{t_{0} \underline{t_{1}} t_{2} \cdots t_{n}}^{j_{1} \cdots j_{p}}\right)\right| j_{1}>0, t_{0}=0, \text { and } t_{1}<-1\right\rangle .
\end{aligned}
$$

Note that, if $j_{1}>0, t_{0}=0$, and $t_{1}>-1$, then

$$
d^{(n-1)}\left(\omega_{t_{0} \underline{t_{1}} t_{2} \cdots t_{n}}^{j_{1} \cdots j_{p}}\right)=\sum_{\ell=1}^{p}(-1)^{\ell+1} \omega_{\underline{(-1)} t_{1} \cdots\left(t_{j_{\ell}}+1\right) \cdots t_{n}}^{1 j_{1} \cdots \hat{j_{\ell} \cdots j_{p}}}=0 .
$$

For $1<i \leq p+1$, we define

$$
\begin{aligned}
W_{i}:= & \left\langle d^{(n-1)}\left(\omega_{t_{0} \underline{t_{1}} t_{2} \cdots t_{n}}^{j_{1} j_{p}}\right)\right| j_{1}=2, \cdots, j_{i-1}=i, \\
& \left.t_{0}=0, t_{1}=\cdots=t_{i-1}=-1, \text { and } t_{i}<-1\right\rangle .
\end{aligned}
$$


Note that

Hence

$$
d^{(n-1)}\left(\omega_{0 \underline{(-1)}}^{23 \cdots(-1) \cdots(-1) t_{p+2} \cdots t_{n}}\right)=0 .
$$

$$
\begin{array}{r}
W_{p+1}:=\left\langle d^{(n-1)}\left(\omega_{t_{0} \underline{t_{1}} t_{2} \cdots t_{n}}^{j_{1} \cdots j_{p}}\right)\right| j_{1}=2, \cdots, j_{p}=p+1, \\
\left.t_{0}=0, t_{1}=\cdots=t_{p}=-1\right\rangle .
\end{array}
$$

If $1<i \leq p$ and $j_{i}>i+1$, then

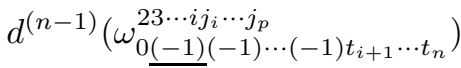

$$
\begin{aligned}
& =\sum_{\ell=i}^{p}(-1)^{i+\ell} d^{(n-1)}\left(\omega_{0 \underline{(-1) \cdots(-1)}\left(23 \cdots i\left(t_{i+1}-1\right) t_{i+2} \cdots t_{j_{\ell-1}}\left(t_{j_{\ell}}+1\right) t_{j_{\ell+1}} \cdots t_{n}\right.}\right) \in W_{i+1} .
\end{aligned}
$$

Therefore

$$
\operatorname{Image}\left(d^{(n-1)}\right)=W_{0}+\cdots+W_{p+1} .
$$

We define

$$
\begin{aligned}
\Omega^{(-1) !} & :=\Omega_{\mathbb{P}^{n} / \kappa}^{p}(m)^{(n)}, \\
\Omega^{i !} & :=\left\langle\underline{\underline{(-1)(-1) \cdots(-1) t_{i+1} \cdots t_{n}}}\right\rangle
\end{aligned}
$$

for $0 \leq i \leq p$. Note that $\Omega^{p !}=W_{p+1}$. We will show that

$$
\Omega^{(i-1) !}=\check{V}_{p, m}^{n-i} \oplus W_{i} \oplus \Omega^{i !}
$$

for $0 \leq i \leq p$, from which the theorem follows easily.

Case $1(i=0)$. If $j_{1}=0$, then

$$
d^{(n-1)}\left(\omega_{t_{0} \underline{t_{1} t_{2} \cdots t_{n}}}^{j_{1} \cdots j_{p}}\right)=\omega_{\underline{t_{0}} t_{1} t_{2} \cdots t_{n}}^{1 j_{1} \cdots j_{p}} .
$$

If $j_{1}>0$ and $t_{0}>0$, then

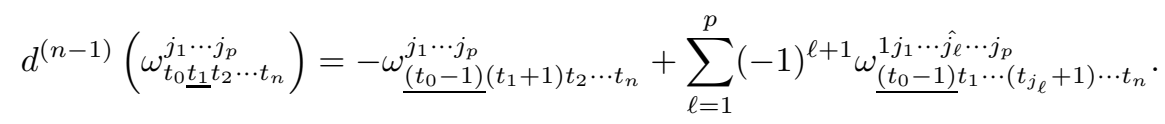

The summation in the above equation is contained in $W_{0}^{\prime}$. Hence

$$
W_{0}=\left\langle\omega_{\underline{t_{0}} t_{1} \cdots j_{n}}^{j_{1} \cdots j_{p}} \mid t_{0} \geq 0\right\rangle .
$$

Recall that

$$
\begin{aligned}
\check{V}_{p, m}^{n} & =\left\langle\omega_{\underline{t_{0}} t_{1} \cdots t_{n}}^{j_{1} \cdots j_{p}} \mid t_{0}<-1\right\rangle, \\
\Omega^{0 !} & =\left\langle\omega_{\underline{t_{0}} t_{1} \cdots t_{n}}^{j_{1} \cdots j_{p}} \mid t_{0}=-1\right\rangle .
\end{aligned}
$$

Therefore

$$
\Omega^{(-1) !}=\check{V}_{p, m}^{n} \oplus W_{0} \oplus \Omega^{0 !} .
$$

Case $2(i=1)$. If $j_{1}>0, t_{0}=0$, and $t_{1}<-1$, then

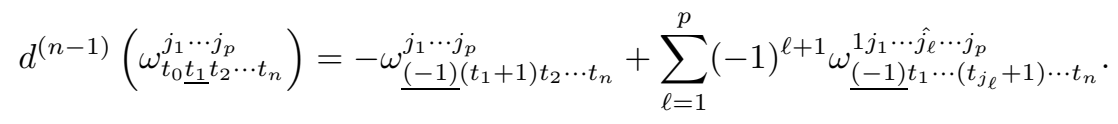

The summation in the above equation is contained in $\check{V}_{p, m}^{n-1}$. Therefore

$$
\Omega^{0 !}=\check{V}_{p, m}^{n-1} \oplus W_{1} \oplus \Omega^{1 !}
$$


Case $3(1<i \leq p)$. If $t_{i}<-1$, then

$$
\begin{aligned}
& d^{(n-1)}\left(\omega_{0 \underline{(-1) \cdots(-1) t_{i} \cdots t_{n}}}^{2 \cdots i j_{i} \cdots j_{p}}\right)=(-1)^{i} \omega^{12 \cdots(i-1) j_{i} \cdots j_{p}} \\
&+\sum_{\ell=i}^{p}(-1)^{\ell+1} \omega^{12 \cdots i j_{i} \cdots \hat{j}_{\ell} \cdots j_{p}} \\
& \underline{(-1) \cdots(-1)\left(t_{i} \cdots\left(t_{j_{\ell}}+1\right) \cdots t_{n}\right.}
\end{aligned}
$$

The summation in the above equation is contained in $\check{V}_{p, m}^{n-i}$; therefore

$$
\Omega^{(i-1) !}=\check{V}_{p, m}^{n-i} \oplus W_{i} \oplus \Omega^{i !}
$$

\section{Corollary 4.9.}

$$
h^{n}\left(\mathbb{P}^{n}, \Omega_{\mathbb{P}^{n} / \kappa}^{p}(m)\right)=\left(\begin{array}{c}
-m-1 \\
n-p
\end{array}\right)\left(\begin{array}{c}
-m+p \\
-m
\end{array}\right) .
$$

Proof. Indeed,

$$
\begin{aligned}
h^{n}\left(\mathbb{P}^{n}, \Omega_{\mathbb{P}^{n} / \kappa}^{p}(m)\right) & =\sum_{i=0}^{p} \operatorname{dim} \check{V}_{p, m}^{n-i} \\
& =\sum_{i=0}^{p}\left(\begin{array}{c}
n-i \\
p-i
\end{array}\right)\left(\begin{array}{c}
-m+p-i-1 \\
n-i
\end{array}\right) \\
& =\left(\begin{array}{c}
-m-1 \\
n-p
\end{array}\right) \sum_{i=0}^{p}\left(\begin{array}{c}
-m+p-i-1 \\
-m-1
\end{array}\right) \\
& =\left(\begin{array}{c}
-m-1 \\
n-p
\end{array}\right)\left(\begin{array}{c}
-m+p \\
-m
\end{array}\right) .
\end{aligned}
$$

\section{REFERENCES}

1. R. Bott. Homogeneous vector bundles. Annals of Math., 66:203-248, 1957. MR 19:681d

2. A. Grothendieck. The cohomology theory of abstract algebraic varieties. In Proc. Internat. Congress Math. (Edinburgh, 1958), pages 103-118. Cambridge University Press, 1960. MR 24:A733

3. R. Hartshorne. Residues and Duality, volume 20 of Lecture Notes in Mathematics. SpringerVerlag, 1966. MR 36:5145

4. R. Hartshorne. Algebraic Geometry. Springer-Verlag, 1977. MR 57:3116

5. I-C. Huang. An explicit construction of residual complexes. Journal of Algebra, 225:698-739, 2000. CMP 2000:09

6. I-C. Huang. Pseudofunctors on modules with zero-dimensional support. Mem. Amer. Math. Soc., 114(no. 548):xii+53, 1995. MR 95h:13013

7. I-C. Huang. A residue map and its applications to some one-dimensional rings. Proc. Amer. Math. Soc., 123(8):2369-2372, 1995. MR 95j:13019

8. I-C. Huang. Residue theorem via an explicit construction of traces. preprint, 1996.

9. I-C. Huang. Theory of residues on the projective plane. Manuscripta Math., 92(2):259-272, 1997. MR 98a:14027

10. R. Hübl. Residues of regular and meromorphic differential forms. Math. Ann., 300(4):605-628, 1994. MR 96e:14017

11. J. Lipman and P. Sastry. Residues and duality for Cousin complexes. preprint, September 21, 1996.

12. P. Sastry. Residues and duality for algebraic schemes. Compositio Math., 101(2):133-178, 1996. MR 98a:14029 
13. P. Sastry and A. Yekutieli. On residue complexes, dualizing sheaves and local cohomology modules. Israel J. Math., 90:325-348, 1995. MR 96f:14021

14. A. Yekutieli. An explicit construction of the Grothendieck residue complex. Astérisque 208, 1992. With an appendix by Pramathanath Sastry. MR 94e:14026

15. A. Yekutieli. Residues and differential operators on schemes. Duke Math. J., 95(2):305-341, 1998. MR 99j:14019

16. A. Yekutieli. Smooth formal embeddings and the residue complex. Canad. J. Math., 50(4):863896, 1998. MR 99i:14004

Institute of Mathematics, Academia Sinica, Nankang, Taipei 11529, Taiwan, R.O.C.

E-mail address: ichuang@math.sinica.edu.tw 